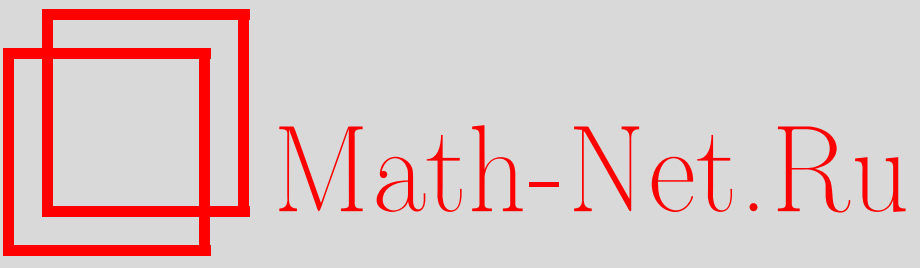

Р. В. Плыкин, Странные аттракторы $A$-каскадов, УМH, 2004, том 59, выпуск 2, 197-198

DOI: https://doi.org/10.4213/rm730

Использование Общероссийского математического портала Math-Net.Ru подразумевает, что вы прочитали и согласны с пользовательским соглашением

http://www.mathnet.ru/rus/agreement

Параметры загрузки:

IP : 54.198 .55 .26

26 апреля 2023 г., 14:56:32 


\title{
СТРАННЫЕ АТТРАКТОРЫ $A$-КАСКАДОВ
}

\author{
Р. В. Плыкин
}

Настоящая заметка является кратким изложением доклада автора на конференции "Колмогоров и современная математика" (Москва, июнь 2003). Изучение стохастических свойств гладких динамических систем зачастую приводит к исследованию устойчивых притягивающих предельных множеств сложной геометрической природы, так называемых "странных аттракторов". Исследуется задача топологической классификации гиперболических странных аттракторов.

\section{1. Квазипорядок Смейла и аттракторы.}

ОПРедЕЛЕнИЕ 1. Считается, что два базисных множества $\Lambda, \Lambda^{\prime} A$-дифффеоморфизма $f$ связаны отношением $\Lambda \succ \Lambda^{\prime}$, если существует цепочка базисных множеств $\Lambda=\Lambda_{1}, \Lambda_{2}, \ldots, \Lambda_{k}=\Lambda^{\prime}$ такая, что $\operatorname{clos} W^{u}\left(\Lambda_{i}\right) \cap \operatorname{clos} W^{s}\left(\Lambda_{i+1}\right) \neq \varnothing$ для $1 \leqslant i \leqslant k-1$. Базисные множества $\Lambda, \Lambda^{\prime}$ считаются эквивалентными $\Lambda \approx \Lambda^{\prime}$, если $\Lambda \succ \Lambda^{\prime} \succ \Lambda$.

ОПРЕДЕЛЕниЕ 2. Отношение $\gamma \succ \gamma^{\prime}$ между двумя классами эквивалентности базисных множеств имеет место, если существуют $\Lambda \in \gamma, \Lambda^{\prime} \in \gamma^{\prime}$, для которых $\Lambda \succ \Lambda^{\prime}$.

Для классов базисных множеств введенное отношение является частичным порядком. При этом каждый класс $\gamma$ состоит из базисных множеств $\Lambda_{1}, \Lambda_{2}, \ldots, \Lambda_{k}$, образующих замкнутую цепочку $\Lambda_{1} \succ \Lambda_{2} \succ \cdots \succ \Lambda_{k} \succ \Lambda_{1}$ по отношению к введенному квазипорядку.

Введем следуюшие обозначения: $W^{s}(\gamma)=\bigcup_{i=1}^{k} W^{s}\left(\Lambda_{i}\right), W^{u}(\gamma)=\bigcup_{i=1}^{k} W^{u}\left(\Lambda_{i}\right),[\gamma]=$ $\bigcup_{i=1}^{k} \Lambda_{i}$.

ОПРеДЕЛЕниЕ 3 . Множество $[\gamma]$, соответствующее минималњному классу эквивалентности базисных множеств относительно квазипорядка Смейла, называется аттрактором. Аттрактор, не являющийся многообразием, назьвается странньм.

Теорема 1. Если ү есть класс эквивалентности базисных мно жеств А-диффеоморфизма $f: M \rightarrow M$ компактного многообразия $M$, то следующие условия әквивалентньь.

I. $\gamma$ есть минимальный класс әквивалентности относительно квазипорядка.

II. $\mathrm{II}_{1}: W^{u}(\gamma)=[\gamma] ; \mathrm{II}_{2}: \gamma$ состоит из единственного базисного множества.

III. Мнохество $W^{s}(\gamma)$ открыто.

IV. Существует такая компактная окрестность $U \supset \operatorname{int} U \supset[\gamma]$, что $f(U) \subset U$ u $\bigcap_{n \geqslant 0} f^{n}(U)=[\gamma]$

ДокАЗАТЕЛЬСТво будет проведено в виде проверки импликаций $\mathrm{I} \Rightarrow \mathrm{II}_{1} \Rightarrow \mathrm{III} \Rightarrow \mathrm{II}_{2} \Rightarrow \mathrm{I}$; $\mathrm{III} \Rightarrow \mathrm{IV} \Rightarrow \mathrm{III}$

$\mathrm{I} \Rightarrow \mathrm{II}_{1}$. Пусть $\gamma$ - минимальньй класс, а $\left\{\gamma_{i}: i=1, \ldots, m\right\}$ - остальные классы эквивалентности базисных множеств. Поскольку $\operatorname{clos} W^{u}(\gamma) \cap \operatorname{clos} W^{s}\left(\gamma_{i}\right)=\varnothing$ для всех $i \in\{1, \ldots, m\}$, существует такое $\varepsilon>0$, что компактная $\varepsilon$-окрестность $B$ множества $\operatorname{clos} W^{u}(\gamma)$ не имеет общих точек с $\bigcup_{i=1}^{m} \operatorname{clos} W^{s}\left(\gamma_{i}\right)$. Рассмотрим множество $F=\bigcap_{n \geqslant 0} f^{n}(B) \supset \operatorname{clos} W^{u}(\gamma)$. Если $x \in F$, то $f^{-n} x \in B$ для всех $n \geqslant 0$, так что $\alpha$ - предельное множество $\alpha(x)$ содержится в $B$ и, следовательно, в $[\gamma]$. Поэтому $x \in W^{u}(\gamma)$. Таким образом, $\operatorname{clos} W^{u}(\gamma) \subset F \subset W^{u}(\gamma)$, откуда вытекает, что $W^{u}(\gamma)$ замкнуто и вследствие $F=\bigcap_{n \geqslant 0} f^{n}(B) \supset W^{u}(\gamma)$ выполнено равенство $F=W^{u}(\gamma)$. Рассмотрим множество $W^{s}(\gamma)$. Согласно теореме о спектралњном разложении $W^{s}(\gamma)=M \backslash \bigcup_{i=1}^{m} W^{s}\left(\gamma_{i}\right) \supset M \backslash \bigcup_{i=1}^{m} \operatorname{clos} W^{s}\left(\gamma_{i}\right) \supset B \supset[\gamma]$. Поэтому для любого $x \in B f^{n}(x) \rightarrow[\gamma]$ при $n \rightarrow+\infty$ и, следовательно, $F=\bigcap_{n \geqslant 0} f^{n}(B)=[\gamma]$, и, таким образом, $[\gamma]=F=W^{u}(\gamma)$.

Для доказательства $\mathrm{II}_{1} \Rightarrow \mathrm{III}$ заметим, что из равенства $[\gamma]=\bigcup_{i=1}^{k} \Lambda_{i}=\bigcup_{i=1}^{k} W^{u}\left(\Lambda_{i}\right)$ вытекают равенства $W^{u}\left(\Lambda_{i}\right)=\Lambda_{i}, i \in\{1, \ldots, k\}$. Утверждение III является следствием локальной структуры произведения базисных множеств. Заметим также, что для любого $\delta>0$ непуста внутренность множеств $W_{\delta}^{s}\left(\Lambda_{i}\right), W_{\delta}^{s}(\gamma)$, составленных локальными диаметра $\delta$ устойчивыми многообразиями точек множеств $\Lambda_{i},[\gamma]$.

Докажем III $\Rightarrow \mathrm{II}_{2}$. Предположим, что в классе $\gamma$ содержатся множества $\Lambda_{i}, i \in\{1, \ldots, k\}$, $\Lambda_{1} \prec \Lambda_{2} \prec \cdots \prec \Lambda_{k} \prec \Lambda_{1}$. Условие $\Lambda_{1} \prec \Lambda_{2}$ эквивалентно непустоте множества $\operatorname{clos} W^{u}\left(\Lambda_{2}\right) \cap$

Работа выполнена при поддержке гранта Президента РФ для поддержки ведущих научных школ № 00-15-96107 и гранта РФФИ-Калуга № 03-01-96303. 
$\operatorname{clos} W^{s}\left(\Lambda_{1}\right)=\Lambda_{2} \cap \operatorname{clos} W^{s}\left(\Lambda_{1}\right)$. Отсюда и из открытости множеств $W^{s}\left(\Lambda_{i}\right)$ следует, что $W^{s}\left(\Lambda_{1}\right) \cap W^{s}\left(\Lambda_{2}\right) \neq \varnothing,-$ приходим к противоречию.

Докажем (II,III) $\Rightarrow$ I. Предположим, что вопреки I найдется класс $\bar{\gamma} \prec \gamma$. Тогда найдутся такие базисные множества $\Lambda \in \gamma, \bar{\Lambda} \in \bar{\gamma}$, что $\operatorname{clos} W^{u}(\Lambda) \cap \operatorname{clos} W^{s}(\bar{\Lambda})=\Lambda \cap \operatorname{clos} W^{s}(\bar{\Lambda}) \neq \varnothing$, откуда вследствие открытости $W^{s}(\Lambda)$ следует непустота множества $W^{s}(\Lambda) \cap W^{s}(\bar{\Lambda})$, что приводит к противоречию.

Таким образом, доказана эквивалентность утверждений I, II и III.

Докажем III $\Leftrightarrow \mathrm{IV}$. Пусть $W^{s}(\gamma)$ - открытое множество, и пусть компактная $\varepsilon$-окрестность $B(\gamma)$ множества $[\gamma]$ входит в $W^{s}(\gamma)$. Тогда $\bigcap_{n \geqslant 0} f^{n} B(\gamma)=[\gamma]$ и согласно одной теореме Смейла существует принадлежащая $B(\gamma)$ компактная окрестность $V \supset[\gamma]$, для которой $f(V) \subset \operatorname{int} V$ и $\bigcap_{n \geqslant 0} f^{n}(V)=[\gamma]$, что и требовалось.

Обратно: пусть существует компактная окрестность $V \supset \operatorname{int} V \supset[\gamma]$, для которой выполнено включение $V \subset f^{-1}$ int $V$. Отсюда следует $\bigcup_{n \geqslant 0} f^{-n}$ int $V \subset W^{s}(\gamma)$. Справедливо и обратное включение $W^{s}(\gamma) \subset \cup f^{-n} \operatorname{int} V$, так как если $x \in W^{s}(\gamma)$, то $f^{n}(x) \rightarrow[\gamma]$ и, следовательно, найдется такое $n \geqslant 0$, что $f^{n}(x) \in \operatorname{int} V$, откуда $x \in f^{-n} \operatorname{int} V \subset \bigcup_{n \geqslant 0} \operatorname{int} f^{-n} V$. Поэтому множество $W^{s}(\gamma)=\bigcup_{n \geqslant 0} f^{-n}$ int $V$ открыто, что и требовалось.

2. О топологической классификации растягивающихся аттракторов коразмерности один.

ОПредЕлЕниЕ 4. Аттрактор $\Lambda \quad A$-каскада $f: M \rightarrow M$, для которого $\operatorname{dim} W^{u}(\Lambda)=$ $\operatorname{dim} M-1$, назьвается растягивающимся аттрактором коразмерности один.

Согласно [1] растягивающиеся аттракторы коразмерности один бывают двух типов: ориентируемые и неориентируемые. Каждьй ориентируемый (неориентируемый) аттрактор порождается парой $(A, P)$ (тройкой $(A, P, \Theta))$, где $A$ - гиперболический автоморфизм тора коразмерности один (характеризующийся тем, что спектр определяемой им унимодулярной матрицы делится единичной окружностью комплексной плоскости на два множества, по крайней мере одно из которых содержит единственный элемент), $P$ - конечное множество периодических траекторий, включающее в себя в случае неориентируемого аттрактора неподвижные точки инволюции $\Theta$ порождающего аттрактор тора, причем $A \Theta=\Theta A$.

ОПредЕЛЕНИЕ 5 . Пусть $A$ - унимодулярная матрица, порождающая аттрактор. Централизатором $Z(A)$ назьвается совокупность унимодулярных, перестановочных с $A$ матриц.

Tеорема 2 (см. [2]). Ориентируемые [неориентируемые] растягивающиеся аттракторы $\Lambda\left(A_{1}, P_{1}\right), \Lambda\left(A_{2}, P_{2}\right) \quad\left[\Lambda\left(A_{1}, P_{1}, \Theta_{1}\right), \Lambda\left(A_{2}, P_{2}, \Theta_{2}\right)\right]$ гомеоморфньи тогда и только тогда, когда существует такое $\Psi$ - линейное отображсение тора вида $\Psi(x)=C x+\xi$ (здесь $C$ - унимодулярная матрица), что $\Psi A_{1} \Psi^{-1} \in Z\left(A_{2}\right)$, при этом $\Psi P_{1}=P_{2}$ $\left[\Theta_{2}=\Psi \Theta_{1} \Psi^{-1}\right]$ и точки спектров матрии, соответствующие одинаковым собственным векторам, одинаково расположены относительно стандартной единичной окружности комплексной плоскости.

В качестве следствия сформулируем следующую теорему.

ТЕОРема 3. Если известно, что ориентируемый [неориентируемый] аттрактор $\Lambda\left(A_{1}, P_{1}\right) \quad\left[\Lambda\left(A_{1}, P_{1}, \Theta_{1}\right)\right]$ порождается гиперболическим автоморфизмом тора коразмерности один с тривиальным иентрализатором, обладающим образующей $A$, то он гомеоморфен ориентируемому [неориентируемому] аттрактору $\Lambda\left(A_{2}, P_{2}\right)$ $\left[\Lambda\left(A_{2}, P_{2}, \Theta_{2}\right)\right]$ тогда и только тогда, когда найдется такое линейное отображение тора $\Psi$, что $\Psi A_{1} \Psi^{-1}=A^{m}, A_{2}=A^{n}, \Psi P_{1}=P_{2} \quad\left[\Theta_{2}=\Psi \Theta_{1} \Psi^{-1}\right]$ и числа $m, n$ одного знака.

\section{СПИСОК ЛИТЕРАТУРЫ}

[1] Р. В. Плыкин // УМН. 1984. Т. 39. №6. С. 75-113. [2] Р. В. Плыкин // УМН. 2002. T. 57. № 6. C. $123-166$.

Обнинский государственный технический университет атомной энергетики
Принято редколлегией 23.01 .2004 